\title{
Effective Cluster Head Election Technique using Multi-Parameter Approach for Wireless Sensor Network
}

\author{
${ }^{1}$ Simran Khiani, ${ }^{2}$ Dr. C. G. Dethe \\ ${ }^{1}$ Asst Professor GHRCEM Pune, \\ ${ }^{2}$ Director, ASC, Nagpur \\ Email:simran.khiani@raisoni.net
}

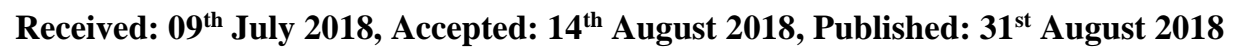

\begin{abstract}
Due to limited resources in wireless sensor network consumption of energy is a challenging issue. In order to improve the network efficiency, a multi parameter approach for selecting cluster head is proposed. The algorithm uses parameters such as remaining energy, distance to Base Station, intra cluster distance and neighboring nodes to select a Cluster Head. The Cluster Head will transfer data to the Base Station using chaining method. The proposed system is more energy aware than the existing systems as per the results.
\end{abstract}

Keywords: Clustering, Energy Aware, Routing, Wireless Sensor Network

\section{Introduction}

Wireless Sensor networks consist of large number of sensor nodes having low power capability, low memory and small size. These sensor nodes are distributed in large areas to observe various environmental parameters such as temperature, moisture, pressure etc. WSN is used in many applications such as object tracking, environment monitoring, military work etc. Due to several constraints such as power of battery, limited memory and limited computation power, designing WSNs for various applications is difficult.

The important factor in sensor nodes is the energy level of battery. As the sensor nodes battery once deployed is not changeable, therefore energy consumption must be optimized. Many protocols have been developed in order to reduce energy consumption. One of the protocols is Hierarchical protocols [1] which use clustering technique.

Clustering [2] method basically divides the network into several groups based on certain parameters. The clustering algorithm has two phases: a) Cluster head selection b) Data transmission. During the initial phase, a node which has maximum energy is chosen as a cluster head. The other nodes in the group are called as member nodes. In the second phase, member nodes sense various parameters such as temperature, pressure, moisture etc. from the environment and collected data is transmitted to the cluster head. The cluster member nodes will switch to idle mode after transmitting the data to the cluster head. It is the cluster head node which will combine all the received data and send it to the Base station for further processing. As the Cluster head only transmits the data to base station, the traffic near the Base station is reduced. Hence energy consumed is also reduced.

Routing Protocols:

Routing is also a very important factor in data transmission. Basically routing is used to select the best path from source node to destination node. In computer network, router is used for routing the data. Each node makes a routing table and then router uses various routing algorithms to select the best path from n number of paths available. The data is then transmitted from source node to destination using the best path. Similarly in Wireless sensor network, the routing protocols are divided into two main categories i.e. protocol operation, network structure.

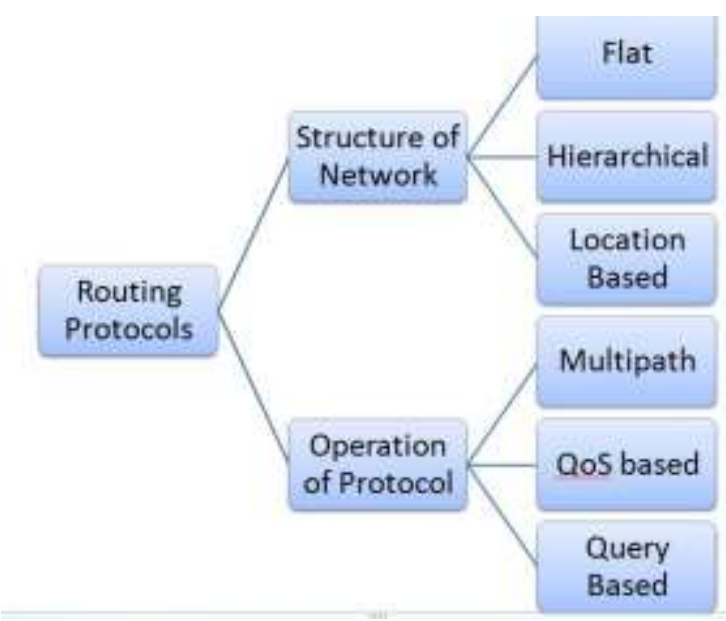

Figure 1: Routing Protocols

Flat routing, all the sensor nodes have same capabilities and perform the sensing task. In Hierarchical routing, a tree like structure is formed and all the nodes are placed at different levels and each node has a different tasks to perform. The nodes at low level perform sensing task and the nodes above the lower level are used for collecting and transmitting data to the sink node or base station.

\section{Clustering}

There are many advantages of using clustering routing method [3] such as scalability, load reduction, robustness, less collisions, less delay. As the Cluster head is aggregating and transmitting the data to the sink node or base station so routing table is not available at each sensor 
node. So this type of routing is easier to manage and it is easy to expand the network to get the values of environmental parameters. Load is also reduced as all other nodes are communicating with the $\mathrm{CH}$. There are less chances of collision and also delay in transmitting the data will also be reduced.

There are different classes in which the clustering is categorized [4]. Clustering method can be centralized, decentralized and hybrid. In centralized method, a central node with higher power will store the details of all other nodes and selection of the path will be decided by that node only. In decentralized method, every node is capable of becoming cluster head in spite of having global information of the network. Hybrid method is combination of both the above methods.

According to the manner in which the sensor nodes work, the clustering algorithms [5] can be divided as Proactive, Reactive and hybrid. In Proactive method, the route to transmit the data is computed in advance before all the data transmission. In reactive method, the route is selected dynamically when data is to be transmitted. Hybrid method is combination of the above two methods. Clustering algorithm can also be classified based on the data transmission that takes place between the nodes in the cluster. The two ways are intra-cluster and intercluster. Intra-cluster method is the communication between sensor node and cluster head whereas Intercluster is the communication between cluster heads. Both the methods can operate in single-hop or multi-hop mode. Depending upon the area of the clusters, the clustering algorithms can also be classified as block based, grid based and chain based. The details about the various algorithms are shown below:

\section{Block based algorithms: \\ LEACH:}

LEACH [6] was developed as the first algorithm in WSNs. LEACH algorithm basically uses signal strength to select a Cluster Head. The $\mathrm{CH}$ forwards the data to the sink node. The major difficulty in LEACH algorithm is it randomly selects $\mathrm{CH}$.

HEED: HEED is a multi- hop clustering algorithm. In this method, the cluster head is selected based on energy and cost of intra cluster communication. Although the HEED method is energy efficient but it has more overhead as lot of packets are broadcasted to select cluster head.

TEEN: TEEN is a clustering routing protocol which focus on the rapid changes in vital applications. In TEEN, there are two thresholds soft and hard. The sensor node will transmit the data only when the sensed value is above the threshold. The problems of HEED algorithm is that it totally dependent on the threshold value, as the sensor will only send the data if it is above the threshold.

Unequal Clustering Size (UCS) [7] algorithm balances the energy consumption of cluster heads. In this method, the network is divided into different layers. Cluster head is a super node which has highest power than other nodes.
The drawback of UCS method is that it should have cluster head at the center of the layer which is not always possible. Hence the system does not balance energy in the network.

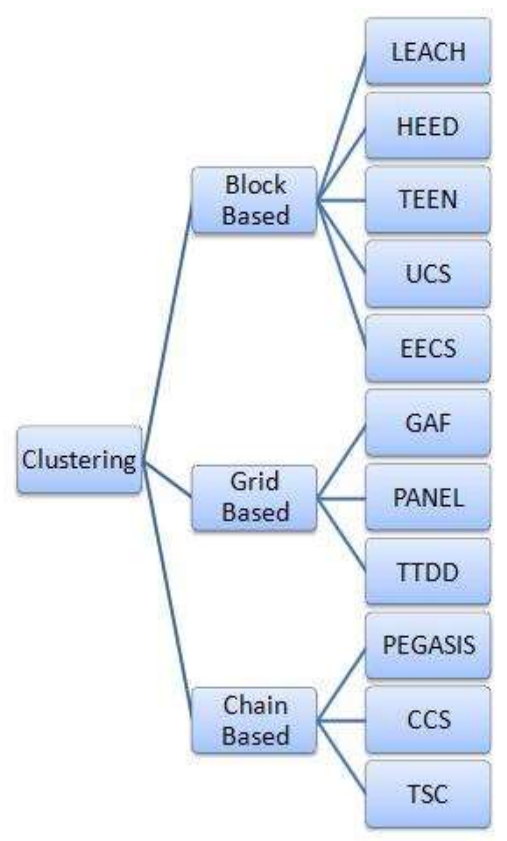

Figure 2: Clustering Algorithms

\section{EECS:}

Energy Efficient Clustering Scheme (EECS) is a single hop clustering method. It is suited for periodic data collection applications. In EECS, the sensor nodes broadcast their energy to the neighboring nodes. The node which is having the maximum residual energy will be appointed as a Cluster head. As the communication between $\mathrm{CH}$ and Base station is single hop so if $\mathrm{CH}$ is at long distance then more energy is consumed.

\section{Grid Based Methods:}

GAF: Geographic Adaptive Fidelity is basically designed for mobile ad hoc networks. It is an energy efficient algorithm based on geographic location. The cells in a cluster coordinate with each other about the active and idle time slots. The best part of algorithm is that one cell in the cluster is always active. The major issues with the algorithm are that the delay is unpredictable and large traffic overhead.

PANEL: Position based aggregator node election protocol which is generally used for database storage applications. This algorithm uses inter-cluster communication to elect a cluster head. This algorithm works well in terms of load balancing, network life time but the disadvantage of the algorithm is that it considers that the clusters are formed during deployment. Hence it is not suitable for real time applications.

TTDD: The two tier dissemination is an algorithm which is scalable and reliable for transmitting data multiple 
sources to multiple and mobile sink nodes. The drawback of this algorithm is it does not uses shortest path to transmit the data hence the algorithm suffers from large latency.

\section{Chain Based clustering algorithms}

PEGASIS [9]: PEGASIS is a clustering algorithm where all the sensor nodes form a chain either by themselves or by the sink node. Pegasis algorithm reduces the amount of data by performing aggregation and also reduces the overhead by forming cluster head. The major concerns in this algorithm are each node must have the information about the complete network and it is difficult to maintain the record of all the nodes.

CCS: Concentric in Clustering Scheme, the network is divided into tracks. The nodes in each circle form a chain. The $\mathrm{CH}$ in each level communicates with the $\mathrm{CH}$ in next level. This reduces the transmission distance and hence the energy consumption. However there are some issues such as routing is based on position of a node not the residual energy which can increase energy holes in the network.

TSC: Track sector clustering is a clustering technique in which the network is divided into tracks and sector. All the levels in each track and sector will have a $\mathrm{CH}$ which reduced the long transmission path in PEGASIS. However the node distribution is not uniform in all the levels and remaining energy is not considered to select a $\mathrm{CH}$.

\section{Proposed System}

In the proposed system, concept of Fuzzy method is applied. The selection of the Cluster head is done by the Base Station as the centralized algorithm works well in all the aspects. The BS has more computing power and large memory capacity than the sensor nodes.

There are many parameters such as remaining energy of the node, neighboring nodes, computing power, distance from BS, location of the node etc. to select Cluster head. However the more prominent parameters which are used in the proposed algorithm are energy, distance from the $\mathrm{BS}$, neighboring nodes, intra-cluster distance.

Initially Base Station will broadcasts a control signal to all the nodes in the network. The sensor nodes will send their information such as energy, distance etc. to the BS. BS after receiving the data from all nodes applies fuzzy method to select a Cluster Head and generates $\mathrm{CH}$ list. The BS send control packet containing $\mathrm{CH}$ details to all the sensor nodes. The sensor nodes then decide which cluster to join depending upon the distance from the $\mathrm{CH}$ and send the control packet containing the information to the $\mathrm{CH}$.

As the network is set, after getting the data from the sensor nodes, the $\mathrm{CHs}$ will first aggregates the data and then form a chain and through the chain the aggregated data is transmitted to the BS.

\section{Parameters}

There are many parameters such as remaining energy, distance to BS, neighbor density, latency, intra-cluster etc. which can be used for selecting $\mathrm{CH}$ [10]. Deciding the parameters for a particular application is also challenging.

In the proposed system, remaining energy of node, distance to the $\mathrm{BS}$, intra-cluster, neighbor nodes are considered for selection purpose.

1. Nodes Energy: The remaining energy of the node plays a very important role as during transmitting and receiving the data, energy is consumed. The range of energy in proposed system is $1 \mathrm{~J}$ to $3 \mathrm{~J}$.

2. Distance to the BS: The location of the BS is also important and it significantly affects the data transmission. For calculating the distance of a node from the BS, Euclidian distance is used. The energy required to transfer data is directly proportional to the distance between the node and BS is increased.

$$
\text { Euclidian Dist. }=\sqrt{ }\left(\mathrm{Xi}_{\mathrm{i}}-\mathrm{Xc}_{\mathrm{c}}\right)^{2}+\left(\mathrm{y}_{\left.\mathrm{i}-\mathrm{y}_{\mathrm{c}}\right)^{2}}\right.
$$

3. Intra-Cluster Distance: It is the distance between the center of the cluster and a node. The average of distance of all the nodes is calculated using the formula:

$$
\begin{aligned}
& \text { IntraCluster. }= \\
& \frac{1}{N} \sum_{1}^{n c} \quad \sum_{1}^{n o s}\|(s i-c i)\|^{2} \\
& \text { Where } \mathrm{n}_{\mathrm{c}}=\text { No. of clusters } \\
& \text { nos=No. of sensors in a cluster } \\
& \mathrm{c}_{\mathrm{s}}=\text { Center of a cluster }
\end{aligned}
$$

4. Inter-Cluster Distance: This distance is calculated from the distance between the various $\mathrm{CHs}$. The minimum distance value is taken into account and accordingly chain of $\mathrm{CHs}$ is formed to transfer data to the BS.

For calculating the inter cluster dist.,the formula used is as follows:

$$
\text { Where } i=1 \text { to } n_{c}-1 \text { and } j=i+1 \text { to } n_{c}
$$

\section{Fuzzy System}

In the proposed system, a type-2 Mamdani FLS (T2MFLS) [11] method is used to select a Cluster Head. The fuzzy system used different components such as fuzzy logic, Rules, inference engine, defuzzifier [12]. The input to the algorithm is energy, neighboring nodes, distance from BS, intra-cluster distance. The output is the probability of node to become $\mathrm{CH}$.

All the input parameters are denoted by levels [13] that is energy is denoted by low, medium, high. The neighboring nodes are denoted by density i.e. Sparse, dense and medium. The distance to the $\mathrm{BS}$ and $\mathrm{CH}$ is denoted by near, medium, far. 27 Rules are applied to these input parameters and the output is probability of a node becoming $\mathrm{CH}$. The table below shows the rules used in Fuzzy logic: 


\begin{tabular}{|c|c|c|c|}
\hline \multicolumn{3}{|c|}{ Input Variables } & \multirow{2}{*}{$\begin{array}{l}\text { Probability to } \\
\text { become } \mathbf{C H}\end{array}$} \\
\hline $\begin{array}{l}\text { Remaining } \\
\text { Energy }\end{array}$ & $\begin{array}{l}\text { Neighbor } \\
\text { Nodes }\end{array}$ & $\begin{array}{l}\text { Distance } \\
\text { to the Base } \\
\text { Station }\end{array}$ & \\
\hline Low & Sparse & Far & Very Weak \\
\hline Low & Sparse & Medium & Weak \\
\hline Low & Sparse & Near & Little Weak \\
\hline High & Dense & Near & Very Strong \\
\hline
\end{tabular}

Algorithm 1 Cluster Head Selection

1: BS sends control packet to all the nodes

2: Apply Fuzzy Logic for $\mathrm{CH}$ selection

3: If node $i$ is alive

4: Call Function Fuzzy Logic

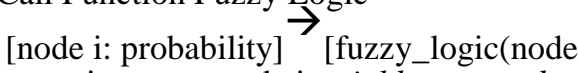
i:energy, node i:neighbours; node $i$ :distance to $B S$, node i: intra-cluster

6: end if distance)

7: For a Cluster, Check the Probability of nodes high_prob $=0.1$

8: for $\mathrm{i}=1: \mathrm{i}<=$ nodecount: $\mathrm{i}++$

9: $\quad$ if [node i:probability] > high_prob] then

10: $\quad$ high_prob=node i:probability

11: node: $i=$ Cluster_Head

12: end if

13: Repeat Same Process for all other Clusters

14: end

Multi-Hop Routing for CHs

After the cluster heads are selected, all member nodes will join the $\mathrm{CH}$ nearby their location[14].The data transmission will take place. All the $\mathrm{CHs}$ will aggregate the data. Then a chain of $\mathrm{CHs}$ will be formed based on the inter cluster distance. The $\mathrm{CH}$ having less inter cluster distance will transmit data to the nearby $\mathrm{CH}$ having less distance. Accordingly the data will be transmitted to the BS.

\section{Results and Conclusion}

The screenshot below shows the result of applying fuzzy based method for $\mathrm{CH}$.

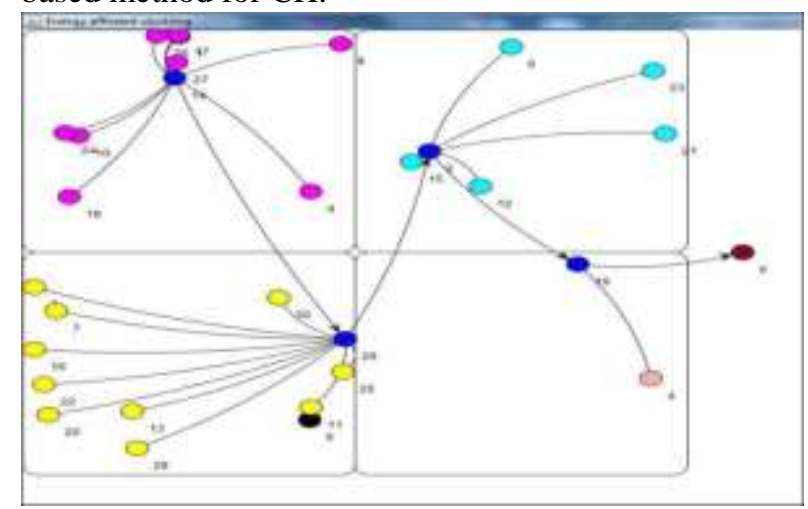

Figure 3: CH Selection and data transmission using Fuzzy method

There are 4 clusters and a $\mathrm{CH}$ is selected based on all the parameters discussed in the proposed method. The nodes with the highest probability is selected as $\mathrm{CH}$ and shown in blue color in figure.

Figure 4 shows the use of four different parameters $\stackrel{(1)}{\rightarrow}$ remaining energy, $\quad \overrightarrow{2}$ distance from the BS, combination and the effect on avg. energy consumption in the network.

When only one parameter is used i.e remaining energy of the node than the average energy consumed is high but when all the four parameters (remaining energy, neighboring nodes, and distance to the BS and intracluster distance) are used for selecting a Cluster head then the energy consumed is less. So, it is clearly seen that when the parameters are increased, the energy consumption in the network is reduced.

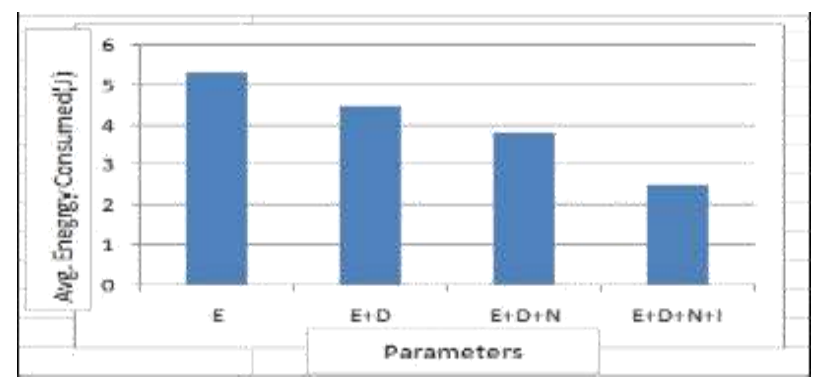

Figure 4:Avg. Energy Consumed by using Different Parameters

The figure below shows the comparison of algorithms LEACH, EEUC, PEGASIS and the proposed algorithm based on half node and first node die concept. FND means the round at which the energy of the first node becomes zero or below threshold and the node is unable to perform any task. Half node die means the round at which half of the nodes in the network is having energy zero or below threshold. The results indicate that as the compared to other algorithms, in the ECHET algorithm HND and FND values are better.

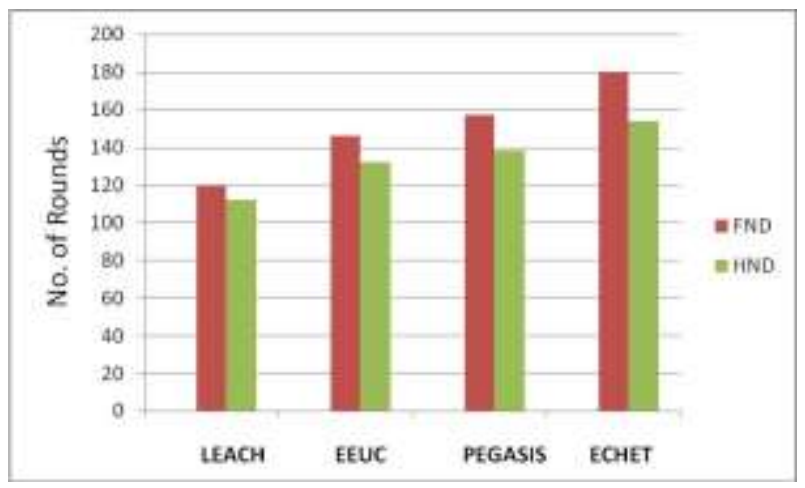

Figure 5: Comparison of Half Node Die (HND) and First Node Die (FND) with Various Algorithms

So the proposed system produces better performance in terms of avg. energy consumption of the network, average energy of nodes than the existing methods. 


\section{References}

1. H. Kiwan, Y. L. Morgan, "Hierarchical Networks: Routing And Clustering (A Concise Survey)," In Proceedings of IEEE CCECE/CCGEI, Ottawa, Canada, 2013.

2. Shreshtha Misra," A Literature Survey on Various Clustering Approaches in Wireless Sensor Network", IEEE 2nd International Conference on Communication, Control and Intelligent Systems (CCIS), 2016.

3. P. Kumarawadu, D. J. Dechene, M. Luccini, and A. Sauer, "Algorithms for node clustering in wireless sensor networks: A survey," in Proceeding 4th International Conference Information Automation Sustainability, Colombo, Sri Lanka, pp. 295-300, December 2008.

4. Xuxun Liu,"Clustering routing algorithms in WSN",KSII Transactions on Internet and Information System Vol 6 no 7, 2012

5. C. Wei, J. Yang,. Y. Gao,Z Zhang., "Cluster-Based Routing Protocols in Wireless Sensor Networks: A Survey" In Proceedings of 2011International Conference on Computer Science and Network Technology, Harbin, China, 24-26 December 2011; pp. 1659-1663.

6. Shreshtha Misra," A Literature Survey on Various Clustering Approaches in Wireless Sensor Network", IEEE 2nd International Conference on Communication, Control and Intelligent Systems (CCIS) 2016.

7. Yong Wang, Changle Li, Yulong Duan," An Energy-Efficient and Swarm Intelligence- Based Routing Protocol for Next-Generation Sensor Networks," Ieee INteLLIGeNt sYstems, 2014.

8. D. J. Dechene, A. El Jardali," Survey of Clustering Algorithms for Wireless Sensor Networks.", Computer Communications. ButterworthHeinemann Newton, MA USA, 2007.

9. Olutayo Boyinbode,"A survey on Clustering routing algorithms", 2013 International conference on network based on Information System.

10. Asif Khan, Israfil Tamim, Emdad Ahmed, Muhammad Abdul Awal," Multiple Parameter Based Clustering (MPC): Prospective Analysis for Effective Clustering in Wireless Sensor Network (WSN) Using K-Means Algorithm”, Wireless Sensor Network, 2012, 4, 18-24

11. Qi-Ye Zhang," A Clustering Routing Protocol for Wireless Sensor Networks Based on Type-2 Fuzzy Logic and ACO”, 2014 IEEE International Conference on Fuzzy Systems (FUZZ-IEEE)

12. Feng Zhang," ICT2TSK: An Improved Clustering Algorithm for WSN Using a Type-2 Takagi-SugenoKang Fuzzy Logic System”, 2013 IEEE

Symposium on Wireless Technology and Applications (ISWTA), September 22-25, 2013, Kuching, Malaysia
13. Ying Zhang,"Netwrok energy efficient based on fuzzy interference system",2017 IEEE

14. Rajesh Purkait," Fuzzy based Unequal Energy Aware Clustering with Multi-hop Routing in Wireless Sensor Network", 2015 IEEE Workshop on Computational Intelligence. 